\title{
Editorial \\ Publisher's Note: We Changed Page Numbers to Article Numbers for Articles Published in Pediatric Reports Volume 1-Volume 12, Issue 2
}

Pediatric Reports Editorial Office

check for updates

Citation: Pediatric Reports Editorial Office. Publisher's Note: We

Changed Page Numbers to Article Numbers for Articles Published in Pediatric Reports Volume 1-Volume 12, Issue 2. Pediatr. Rep. 2022, 14, 93-98. https://doi.org/10.3390/ pediatric14010014

Received: 22 February 2022 Accepted: 22 February 2022 Published: 23 February 2022

Publisher's Note: MDPI stays neutral with regard to jurisdictional claims in published maps and institutional affiliations.

Copyright: (C) 2022 by the author. Licensee MDPI, Basel, Switzerland. This article is an open access article distributed under the terms and conditions of the Creative Commons Attribution (CC BY) license (https:// creativecommons.org/licenses/by/ $4.0 /)$.
MDPI AG, St. Alban-Anlage 66, 4052 Basel, Switzerland; pediatrrep@mdpi.com

Pediatric Reports [1] was published by PAGEPress from Volume 1 (2009) to Volume 12, Issue 2 (2020). From Volume 12, Issue 3 (2020), Pediatric Reports is published by MDPI.

Previous articles in Volume 1-Volume 12, Issue 2, published by PAGEPress in Open Access under a CC-BY (or CC-BY-NC-ND) licence, are now hosted by MDPI on mdpi.com as a courtesy and upon agreement with PAGEPress.

To standardize the metadata format of all previous articles, MDPI republished 245 articles of Volume 1-Volume 12, Issue 2 with article numbers replacing page numbers (Table 1). MDPI also corrected Issue Number errors that occurred during importing of batch data for 24 articles in Volumes 1-5 and 7 (Table 2).

Table 1. MDPI changed page numbers to articles numbers for 275 articles in Volume 1-Volume 12, Issue 2.

\begin{tabular}{ccc}
\hline DOI & Previous Page Number & Current Article Number \\
\hline $10.4081 /$ pr.2009.e1 & $1-9$ & e1 \\
$10.4081 /$ pr.2009.e2 & $10-12$ & e2 \\
$10.4081 /$ pr.2009.e3 & $13-15$ & e3 \\
$10.4081 /$ pr.2009.e4 & $16-18$ & e4 \\
$10.4081 /$ pr.2009.e6 & $20-22$ & e6 \\
$10.4081 /$ pr.2009.e7 & $23-26$ & e7 \\
$10.4081 /$ pr.2009.e8 & $27-29$ & e8 \\
$10.4081 /$ pr.2009.e9 & $30-31$ & e9 \\
$10.4081 /$ pr.2010.e1 & $1-8$ & e1 \\
$10.4081 /$ pr.2010.e2 & $9-10$ & e2 \\
$10.4081 /$ pr.2010.e3 & $11-14$ & e3 \\
$10.4081 /$ pr.2010.e4 & $15-17$ & e4 \\
$10.4081 /$ pr.2010.e5 & $18-19$ & e5 \\
$10.4081 /$ pr.2010.e6 & $20-21$ & e6 \\
$10.4081 /$ pr.2010.e7 & $22-25$ & e7 \\
$10.4081 /$ pr.2010.e8 & $26-28$ & e8 \\
$10.4081 /$ pr.2010.e9 & $29-31$ & e9 \\
$10.4081 /$ pr.2010.e10 & $32-35$ & e10 \\
$10.4081 /$ pr.2010.e11 & $36-37$ & e11 \\
$10.4081 /$ pr.2010.e12 & $38-41$ & e12 \\
$10.4081 /$ pr.2010.e13 & $42-43$ & e13 \\
$10.4081 /$ pr.2010.e14 & $44-47$ & e14 \\
$10.4081 /$ pr.2010.e15 & $48-50$ & e15 \\
$10.4081 /$ pr.2010.e16 & $51-54$ & e16 \\
$10.4081 /$ pr.2010.e17 & $55-57$ & e17 \\
$10.4081 /$ pr.2010.e18 & $58-61$ & e18 \\
\hline
\end{tabular}


Table 1. Cont.

\begin{tabular}{|c|c|c|}
\hline DOI & Previous Page Number & Current Article Number \\
\hline 10.4081/pr.2010.e19 & $62-66$ & e19 \\
\hline 10.4081/pr.2010.e20 & $67-68$ & e20 \\
\hline 10.4081/pr.2011.e1 & $1-5$ & e1 \\
\hline 10.4081/pr.2011.e2 & $6-8$ & e2 \\
\hline 10.4081/pr.2011.e3 & $9-10$ & e3 \\
\hline 10.4081/pr.2011.e4 & $11-12$ & e4 \\
\hline 10.4081/pr.2011.e5 & $13-16$ & e5 \\
\hline 10.4081/pr.2011.e6 & $17-20$ & e6 \\
\hline 10.4081/pr.2011.e7 & $21-24$ & e7 \\
\hline 10.4081/pr.2011.e8 & $25-26$ & e8 \\
\hline 10.4081/pr.2011.e9 & $27-30$ & e9 \\
\hline 10.4081/pr.2011.e10 & $31-36$ & e10 \\
\hline 10.4081/pr.2011.e11 & $37-38$ & e11 \\
\hline 10.4081/pr.2011.e12 & $39-41$ & e12 \\
\hline 10.4081/pr.2011.e13 & $42-44$ & e13 \\
\hline 10.4081/pr.2011.e14 & $45-48$ & e14 \\
\hline 10.4081/pr.2011.e15 & $49-50$ & e15 \\
\hline 10.4081/pr.2011.e16 & $51-64$ & e16 \\
\hline 10.4081/pr.2011.e17 & $65-78$ & e17 \\
\hline 10.4081/pr.2011.e18 & $79-80$ & e18 \\
\hline 10.4081/pr.2011.e19 & $81-83$ & e19 \\
\hline 10.4081/pr.2011.e20 & $84-86$ & $\mathrm{e} 20$ \\
\hline 10.4081/pr.2011.e21 & $87-90$ & e21 \\
\hline 10.4081/pr.2011.e22 & $91-92$ & $\mathrm{e} 22$ \\
\hline 10.4081/pr.2011.e23 & $93-96$ & $\mathrm{e} 23$ \\
\hline 10.4081/pr.2011.e24 & 97-99 & $\mathrm{e} 24$ \\
\hline 10.4081/pr.2011.e26 & $103-107$ & $\mathrm{e} 26$ \\
\hline 10.4081/pr.2011.e27 & $108-110$ & $\mathrm{e} 27$ \\
\hline 10.4081/pr.2011.e28 & $111-113$ & e28 \\
\hline 10.4081/pr.2011.e29 & $114-118$ & e29 \\
\hline 10.4081/pr.2011.e30 & 119-121 & e30 \\
\hline 10.4081/pr.2011.e31 & $122-124$ & e31 \\
\hline 10.4081/pr.2011.e32 & $125-126$ & e32 \\
\hline 10.4081/pr.2011.e33 & $127-128$ & e33 \\
\hline 10.4081/pr.2011.e34 & $129-132$ & e34 \\
\hline 10.4081/pr.2011.s2.e1 & $1-2$ & e1 \\
\hline 10.4081/pr.2011.s2.e2 & 3 & e2 \\
\hline 10.4081/pr.2011.s2.e3 & 4 & e3 \\
\hline 10.4081/pr.2011.s2.e4 & $5-7$ & e4 \\
\hline 10.4081/pr.2011.s2.e5 & $8-10$ & e5 \\
\hline 10.4081/pr.2011.s2.e6 & $11-13$ & e6 \\
\hline 10.4081/pr.2011.s2.e7 & $14-17$ & e7 \\
\hline 10.4081/pr.2011.s2.e8 & $18-20$ & e8 \\
\hline 10.4081/pr.2011.s2.e9 & $21-24$ & e9 \\
\hline 10.4081/pr.2011.s2.e10 & $25-27$ & e10 \\
\hline 10.4081/pr.2011.s2.e11 & $28-31$ & e11 \\
\hline 10.4081/pr.2011.s2.e12 & $32-33$ & e12 \\
\hline 10.4081/pr.2011.s2.e13 & $34-37$ & e13 \\
\hline 10.4081/pr.2011.s2.e14 & $38-42$ & e14 \\
\hline 10.4081/pr.2011.s2.e15 & $43-47$ & e15 \\
\hline 10.4081/pr.2012.e1 & $1-4$ & e1 \\
\hline 10.4081/pr.2012.e2 & $5-9$ & e2 \\
\hline 10.4081/pr.2012.e3 & $10-11$ & e3 \\
\hline 10.4081/pr.2012.e4 & $12-13$ & e4 \\
\hline 10.4081/pr.2012.e5 & $14-16$ & e5 \\
\hline 10.4081/pr.2012.e6 & $17-20$ & e6 \\
\hline
\end{tabular}


Table 1. Cont.

\begin{tabular}{|c|c|c|}
\hline DOI & Previous Page Number & Current Article Number \\
\hline 10.4081/pr.2012.e7 & $21-24$ & e7 \\
\hline 10.4081/pr.2012.e8 & $25-29$ & e8 \\
\hline $10.4081 /$ pr.2012.e9 & $30-33$ & e9 \\
\hline 10.4081/pr.2012.e10 & $34-37$ & e10 \\
\hline 10.4081/pr.2012.e11 & $38-43$ & e11 \\
\hline 10.4081/pr.2012.e12 & $44-47$ & e12 \\
\hline $10.4081 /$ pr.2012.e13 & $48-51$ & $\mathrm{e} 13$ \\
\hline 10.4081/pr.2012.e14 & $52-53$ & e14 \\
\hline 10.4081/pr.2012.e15 & $54-56$ & e15 \\
\hline $10.4081 /$ pr.2012.e16 & $57-63$ & e16 \\
\hline 10.4081/pr.2012.e17 & $64-67$ & e17 \\
\hline 10.4081/pr.2012.e18 & $68-69$ & e18 \\
\hline 10.4081/pr.2012.e19 & $70-77$ & e19 \\
\hline 10.4081/pr.2012.e20 & $78-81$ & e20 \\
\hline 10.4081/pr.2012.e21 & $82-84$ & e21 \\
\hline 10.4081/pr.2012.e22 & 85 & $\mathrm{e} 22$ \\
\hline 10.4081/pr.2012.e23 & $86-87$ & $\mathrm{e} 23$ \\
\hline 10.4081/pr.2012.e24 & $88-90$ & $\mathrm{e} 24$ \\
\hline 10.4081/pr.2012.e25 & $91-93$ & e25 \\
\hline 10.4081/pr.2012.e26 & $94-96$ & $\mathrm{e} 26$ \\
\hline 10.4081/pr.2012.e27 & 97 & e27 \\
\hline 10.4081/pr.2012.e28 & $98-100$ & e28 \\
\hline 10.4081/pr.2012.e29 & $101-104$ & e29 \\
\hline 10.4081/pr.2012.e30 & $105-107$ & e30 \\
\hline 10.4081/pr.2012.e31 & $108-111$ & e31 \\
\hline 10.4081/pr.2012.e32 & $112-114$ & e32 \\
\hline 10.4081/pr.2012.e33 & $115-116$ & $\mathrm{e} 33$ \\
\hline 10.4081/pr.2012.e34 & $117-118$ & e34 \\
\hline 10.4081/pr.2012.e35 & $119-123$ & e35 \\
\hline 10.4081/pr.2012.e36 & $124-126$ & e36 \\
\hline 10.4081/pr.2012.e37 & $127-129$ & e37 \\
\hline 10.4081/pr.2013.e1 & $1-7$ & e1 \\
\hline 10.4081/pr.2013.e2 & $8-12$ & e2 \\
\hline $10.4081 /$ pr.2013.e3 & $13-16$ & e3 \\
\hline 10.4081/pr.2013.e4 & $17-19$ & e4 \\
\hline $10.4081 /$ pr.2013.e5 & $20-23$ & e5 \\
\hline $10.4081 /$ pr.2013.e6 & $24-27$ & e6 \\
\hline $10.4081 /$ pr.2013.e7 & $28-30$ & $\mathrm{e} 7$ \\
\hline 10.4081/pr.2013.e8 & $31-34$ & e8 \\
\hline 10.4081/pr.2013.e9 & $35-37$ & e9 \\
\hline 10.4081/pr.2013.e10 & $38-42$ & e10 \\
\hline 10.4081/pr.2013.e11 & $43-47$ & e11 \\
\hline 10.4081/pr.2013.e12 & $48-49$ & $\mathrm{e} 12$ \\
\hline 10.4081/pr.2013.e13 & $51-52$ & e13 \\
\hline 10.4081/pr.2013.e14 & $53-57$ & e14 \\
\hline 10.4081/pr.2013.e15 & $58-63$ & e15 \\
\hline 10.4081/pr.2013.e16 & $64-72$ & e16 \\
\hline 10.4081/pr.2013.e17 & $73-75$ & e17 \\
\hline 10.4081/pr.2013.e18 & $76-80$ & e18 \\
\hline 10.4081/pr.2013.e19 & $81-85$ & e19 \\
\hline $10.4081 /$ pr.2014.5090 & $26-28$ & 5090 \\
\hline $10.4081 /$ pr.2014.5112 & $23-25$ & 5112 \\
\hline 10.4081/pr.2014.5118 & $1-7$ & 5118 \\
\hline 10.4081/pr.2014.5126 & $8-11$ & 5126 \\
\hline $10.4081 /$ pr.2014.5160 & $31-32$ & 5160 \\
\hline $10.4081 /$ pr.2014.5186 & $19-22$ & 5186 \\
\hline 10.4081/pr.2014.5194 & $12-15$ & 5194 \\
\hline $10.4081 /$ pr.2014.5311 & $16-18$ & 5311 \\
\hline
\end{tabular}


Table 1. Cont.

\begin{tabular}{|c|c|c|}
\hline DOI & Previous Page Number & Current Article Number \\
\hline 10.4081/pr.2014.5332 & $29-30$ & 5332 \\
\hline 10.4081/pr.2014.5368 & $33-36$ & 5368 \\
\hline 10.4081/pr.2014.5447 & $37-39$ & 5447 \\
\hline $10.4081 /$ pr.2014.5491 & $49-50$ & 5491 \\
\hline 10.4081/pr.2014.5532 & $40-43$ & 5532 \\
\hline $10.4081 /$ pr.2014.5534 & $44-48$ & 5534 \\
\hline 10.4081/pr.2014.5596 & $51-52$ & 5596 \\
\hline 10.4081/pr.2014.5619 & $53-55$ & 5619 \\
\hline $10.4081 /$ pr.2014.5660 & $56-60$ & 5660 \\
\hline 10.4081/pr.2015.5578 & $16-18$ & 5578 \\
\hline $10.4081 /$ pr.2015.5615 & $7-12$ & 5615 \\
\hline 10.4081/pr.2015.5659 & $1-6$ & 5659 \\
\hline $10.4081 /$ pr.2015.5682 & $13-15$ & 5682 \\
\hline 10.4081/pr.2015.5703 & $22-27$ & 5703 \\
\hline 10.4081/pr.2015.5760 & $19-21$ & 5760 \\
\hline 10.4081/pr.2015.5795 & $31-34$ & 5795 \\
\hline 10.4081/pr.2015.5817 & $74-75$ & 5817 \\
\hline 10.4081/pr.2015.5858 & $45-47$ & 5858 \\
\hline 10.4081/pr.2015.5859 & $28-30$ & 5859 \\
\hline $10.4081 /$ pr.2015.5872 & $38-44$ & 5872 \\
\hline 10.4081/pr.2015.5873 & $51-53$ & 5873 \\
\hline 10.4081/pr.2015.5914 & $54-55$ & 5914 \\
\hline 10.4081/pr.2015.5928 & $62-64$ & 5928 \\
\hline 10.4081/pr.2015.5936 & $48-50$ & 5936 \\
\hline 10.4081/pr.2015.5938 & $56-58$ & 5938 \\
\hline 10.4081/pr.2015.5951 & $76-78$ & 5951 \\
\hline 10.4081/pr.2015.5955 & $35-37$ & 5955 \\
\hline 10.4081/pr.2015.5999 & $59-61$ & 5999 \\
\hline 10.4081/pr.2015.6056 & $65-70$ & 6056 \\
\hline 10.4081/pr.2015.6228 & $72-73$ & 6228 \\
\hline 10.4081/pr.2015.6338 & $79-80$ & 6338 \\
\hline 10.4081/pr.2016.6112 & $15-20$ & 6112 \\
\hline 10.4081/pr.2016.6159 & $1-3$ & 6159 \\
\hline 10.4081/pr.2016.6188 & $12-14$ & 6188 \\
\hline 10.4081/pr.2016.6370 & $21-26$ & 6370 \\
\hline $10.4081 /$ pr.2016.6395 & $4-5$ & 6395 \\
\hline 10.4081/pr.2016.6456 & $9-11$ & 6456 \\
\hline 10.4081/pr.2016.6487 & $42-45$ & 6487 \\
\hline 10.4081/pr.2016.6488 & $30-33$ & 6488 \\
\hline 10.4081/pr.2016.6516 & $34-38$ & 6516 \\
\hline $10.4081 /$ pr.2016.6517 & $39-41$ & 6517 \\
\hline 10.4081/pr.2016.6555 & $46-47$ & 6555 \\
\hline 10.4081/pr.2016.6576 & $68-70$ & 6576 \\
\hline 10.4081/pr.2016.6596 & $27-29$ & 6596 \\
\hline $10.4081 /$ pr.2016.6613 & $74-76$ & 6613 \\
\hline 10.4081/pr.2016.6615 & $50-52$ & 6615 \\
\hline 10.4081/pr.2016.6626 & $48-49$ & 6626 \\
\hline 10.4081/pr.2016.6632 & $53-58$ & 6632 \\
\hline 10.4081/pr.2016.6643 & $61-62$ & 6643 \\
\hline 10.4081/pr.2016.6685 & $63-67$ & 6685 \\
\hline 10.4081/pr.2016.6885 & $71-73$ & 6885 \\
\hline 10.4081/pr.2017.6810 & $14-15$ & 6810 \\
\hline 10.4081/pr.2017.6946 & $1-3$ & 6946 \\
\hline 10.4081/pr.2017.6962 & $10-13$ & 6962 \\
\hline 10.4081/pr.2017.6973 & $7-9$ & 6973 \\
\hline 10.4081/pr.2017.6984 & $4-6$ & 6984 \\
\hline 10.4081/pr.2017.7045 & $33-35$ & 7045 \\
\hline
\end{tabular}


Table 1. Cont.

\begin{tabular}{|c|c|c|}
\hline DOI & Previous Page Number & Current Article Number \\
\hline 10.4081/pr.2017.7084 & $17-20$ & 7084 \\
\hline 10.4081/pr.2017.7085 & $50-52$ & 7085 \\
\hline 10.4081/pr.2017.7163 & $29-32$ & 7163 \\
\hline 10.4081/pr.2017.7211 & $26-28$ & 7211 \\
\hline 10.4081/pr.2017.7214 & $21-25$ & 7214 \\
\hline 10.4081/pr.2017.7227 & $70-77$ & 7227 \\
\hline 10.4081/pr.2017.7240 & $64-69$ & 7240 \\
\hline 10.4081/pr.2017.7266 & $36-39$ & 7266 \\
\hline 10.4081/pr.2017.7270 & $53-54$ & 7270 \\
\hline $10.4081 /$ pr.2017.7284 & $42-46$ & 7284 \\
\hline 10.4081/pr.2017.7294 & $58-63$ & 7294 \\
\hline $10.4081 /$ pr.2017.7300 & $40-41$ & 7300 \\
\hline 10.4081/pr.2017.7301 & $47-49$ & 7301 \\
\hline 10.4081/pr.2017.7305 & $55-57$ & 7305 \\
\hline 10.4081/pr.2017.7341 & $78-80$ & 7341 \\
\hline 10.4081/pr.2017.7489 & $84-87$ & 7489 \\
\hline 10.4081/pr.2018.7232 & $17-23$ & 7232 \\
\hline 10.4081/pr.2018.7339 & $1-7$ & 7339 \\
\hline $10.4081 /$ pr.2018.7424 & $11-13$ & 7424 \\
\hline 10.4081/pr.2018.7500 & $29-32$ & 7500 \\
\hline 10.4081/pr.2018.7514 & $14-16$ & 7514 \\
\hline 10.4081/pr.2018.7604 & $24-25$ & 7604 \\
\hline 10.4081/pr.2018.7636 & $46-48$ & 7636 \\
\hline 10.4081/pr.2018.7648 & $33-36$ & 7648 \\
\hline 10.4081/pr.2018.7689 & $37-38$ & 7689 \\
\hline 10.4081/pr.2018.7705 & $28-42$ & 7705 \\
\hline 10.4081/pr.2018.7752 & $43-45$ & 7752 \\
\hline 10.4081/pr.2019.7386 & $6-10$ & 7386 \\
\hline 10.4081/pr.2019.7839 & $1-5$ & 7839 \\
\hline 10.4081/pr.2019.7848 & $31-34$ & 7848 \\
\hline 10.4081/pr.2019.7951 & $21-24$ & 7951 \\
\hline 10.4081/pr.2019.7953 & $13-14$ & 7953 \\
\hline 10.4081/pr.2019.7954 & $15-20$ & 7954 \\
\hline 10.4081/pr.2019.7973 & $11-12$ & 7973 \\
\hline 10.4081/pr.2019.7993 & $35-37$ & 7993 \\
\hline 10.4081/pr.2019.7997 & $25-30$ & 7997 \\
\hline 10.4081/pr.2019.8029 & $64-71$ & 8029 \\
\hline 10.4081/pr.2019.8161 & $41-43$ & 8161 \\
\hline 10.4081/pr.2019.8165 & $44-48$ & 8165 \\
\hline 10.4081/pr.2019.8166 & $56-58$ & 8166 \\
\hline 10.4081/pr.2019.8184 & $38-40$ & 8184 \\
\hline 10.4081/pr.2019.8190 & $49-52$ & 8190 \\
\hline 10.4081/pr.2019.8206 & $53-55$ & 8206 \\
\hline 10.4081/pr.2019.8224 & $78-80$ & 8224 \\
\hline 10.4081/pr.2019.8248 & $75-77$ & 8248 \\
\hline 10.4081/pr.2019.8250 & $72-74$ & 8250 \\
\hline 10.4081/pr.2019.8251 & $59-63$ & 8251 \\
\hline 10.4081/pr.2020.7405 & $3-6$ & 7405 \\
\hline 10.4081/pr.2020.7998 & $7-13$ & 7998 \\
\hline 10.4081/pr.2020.8231 & $20-21$ & 8231 \\
\hline 10.4081/pr.2020.8254 & 14-19 & 8254 \\
\hline 10.4081/pr.2020.8352 & $35-38$ & 8352 \\
\hline 10.4081/pr.2020.8382 & $29-33$ & 8382 \\
\hline 10.4081/pr.2020.8430 & $42-45$ & 8430 \\
\hline 10.4081/pr.2020.8476 & $26-28$ & 8476 \\
\hline 10.4081/pr.2020.8483 & $39-41$ & 8483 \\
\hline 10.4081/pr.2020.8486 & $22-25$ & 8486 \\
\hline 10.4081/pr.2020.8495 & $1-2$ & 8495 \\
\hline
\end{tabular}


Table 1. Cont.

\begin{tabular}{ccc}
\hline DOI & Previous Page Number & Current Article Number \\
\hline $10.4081 /$ pr.2020.8505 & $50-55$ & 8505 \\
$10.4081 /$ pr.2020.8535 & $56-60$ & 8535 \\
$10.4081 /$ pr.2020.8595 & $61-63$ & 8595 \\
$10.4081 /$ pr.2020.8690 & $1-3$ & 8690 \\
$10.4081 /$ pr.2020.8691 & $4-7$ & 8691 \\
$10.4081 /$ pr.2020.8692 & $8-10$ & 8692 \\
$10.4081 /$ pr.2020.8693 & $11-14$ & 8693 \\
$10.4081 /$ pr.2020.8694 & $15-17$ & 8694 \\
$10.4081 /$ pr.2020.8695 & $18-22$ & 8695 \\
$10.4081 /$ pr.2020.8696 & $23-26$ & 8696 \\
$10.4081 /$ pr.2020.8697 & $27-29$ & 8697 \\
$10.4081 /$ pr.2020.8698 & $30-33$ & 8698 \\
$10.4081 /$ pr.2020.8699 & $34-38$ & 8699 \\
$10.4081 /$ pr.2020.8700 & $39-43$ & 8700 \\
$10.4081 /$ pr.2020.8701 & $44-46$ & 8701 \\
$10.4081 /$ pr.2020.8702 & $47-51$ & 8702 \\
$10.4081 /$ pr.2020.8703 & $52-56$ & 8703 \\
$10.4081 /$ pr.2020.8758 & 1 & 8758 \\
$10.4081 /$ pr.2020.8823 & $64-66$ & 8823 \\
\hline
\end{tabular}

Table 2. MDPI corrected Issue numbers for 24 articles in Volumes 1-5 and 7.

\begin{tabular}{ccc}
\hline DOI & Previous Issue Number & Current Issue Number \\
\hline $10.4081 / 3929$ & 13 & $\mathrm{~s} 3$ \\
$10.4081 / 4585$ & 12 & $\mathrm{~s} 2$ \\
$10.4081 /$ pr.2009.s1 & 11 & $\mathrm{~s} 1$ \\
$10.4081 /$ pr.2010.s1 & 11 & $\mathrm{~s} 1$ \\
$10.4081 /$ pr.2011.s1 & 11 & $\mathrm{~s} 1$ \\
$10.4081 /$ pr.2011.s2.e1 & 12 & $\mathrm{~s} 2$ \\
$10.4081 /$ pr.2011.s2.e2 & 12 & $\mathrm{~s} 2$ \\
$10.4081 /$ pr.2011.s2.e3 & 12 & $\mathrm{~s} 2$ \\
$10.4081 /$ pr.2011.s2.e4 & 12 & $\mathrm{~s} 2$ \\
$10.4081 /$ pr.2011.s2.e5 & 12 & $\mathrm{~s} 2$ \\
$10.4081 /$ pr.2011.s2.e6 & 12 & $\mathrm{~s} 2$ \\
$10.4081 /$ pr.2011.s2.e7 & 12 & $\mathrm{~s} 2$ \\
$10.4081 /$ pr.2011.s2.e8 & 12 & $\mathrm{~s} 2$ \\
$10.4081 /$ pr.2011.s2.e9 & 12 & $\mathrm{~s} 2$ \\
$10.4081 /$ pr.2011.s2.e10 & 12 & $\mathrm{~s} 2$ \\
$10.4081 /$ pr.2011.s2.e11 & 12 & $\mathrm{~s} 2$ \\
$10.4081 /$ pr.2011.s2.e12 & 12 & $\mathrm{~s} 2$ \\
$10.4081 /$ pr.2011.s2.e13 & 12 & $\mathrm{~s} 2$ \\
$10.4081 /$ pr.2011.s2.e14 & 12 & $\mathrm{~s} 2$ \\
$10.4081 /$ pr.2011.s2.e15 & 12 & $\mathrm{~s} 2$ \\
$10.4081 / 2012 . s 1 . a b s$ & 11 & $\mathrm{~s} 1$ \\
$10.4081 / 2012 . s 1 . r e l$ & 11 & $\mathrm{~s} 2$ \\
$10.4081 / 2013 . s 1 . a b s$ & 11 & $\mathrm{~s} 1$ \\
$10.4081 / 2013 . s 1 . r e l$ & 11 & $\mathrm{~s} 1$ \\
$10.4081 /$ pr.2015.6202 & 11 & $\mathrm{~s} 1$ \\
\hline
\end{tabular}

Conflicts of Interest: The author declares no conflict of interest.

\section{Reference}

1. Pediatric Reports Homepage. Available online: https://www.mdpi.com/journal/pediatrrep (accessed on 10 February 2022). 